Revue des patrimoines

$37 \mid 2018$

Jardins collectifs : de l'abbé Lemire aux jardins d'insertion. Typologies - Expériences - Enjeux de conservation

\title{
Jardins familiaux, jardins partagés à Bordeaux entre alimentation et multifonctionnalités
}

Family gardens and shared gardens in Bordeaux, from providing food to other, multiple uses

\section{Véronique Saint-Ges}

\section{(2) OpenEdition}

\section{Journals}

Édition électronique

URL : http://journals.openedition.org/insitu/18956

DOI : 10.4000/insitu.18956

ISSN : 1630-7305

Éditeur

Ministère de la Culture

Référence électronique

Véronique Saint-Ges, « Jardins familiaux, jardins partagés à Bordeaux entre alimentation et multifonctionnalités », In Situ [En ligne], 37 | 2018, mis en ligne le 13 décembre 2018, consulté le 15 novembre 2019. URL : http://journals.openedition.org/insitu/18956 ; DOI : 10.4000/insitu.18956

Ce document a été généré automatiquement le 15 novembre 2019.

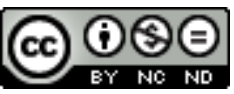

In Situ Revues des patrimoines est mis à disposition selon les termes de la licence Creative Commons Attribution - Pas d'Utilisation Commerciale - Pas de Modification 4.0 International. 


\section{Jardins familiaux, jardins partagés à Bordeaux entre alimentation et multifonctionnalités}

Family gardens and shared gardens in Bordeaux, from providing food to other, multiple uses

Véronique Saint-Ges

1 Les jardins, qu'ils soient familiaux ou partagés, héritage des jardins ouvriers du XIX siècle, suscitent un nouvel engouement au niveau mondial, dans de multiples métropoles (Détroit, Montréal, Paris, Todmorden ${ }^{1}$, etc.) et pays (ex: projet «10000 jardins en Afrique »). Porteurs d'enjeux sociaux, économiques et environnementaux, les jardins collectifs possèdent de nombreuses fonctionnalités : fonction paysagère et nourricière, fonction environnementale en revitalisant les sols d'espaces urbains désaffectés, fonction éducative à l'agriculture et à une alimentation plus saine, fonction sociale par la réinsertion de personnes en difficulté en les aidant à reconstruire des liens sociaux, ou en permettant aux faibles revenus de se nourrir avec une nourriture de qualité, etc. Cette énumération montre combien les enjeux portés par les jardins familiaux, forme la plus ancienne de l'agriculture urbaine ${ }^{2}$, sont nombreux. En effet, les crises alimentaires diverses, la demande forte des urbains pour une meilleure qualité de vie et alimentation font que l'agriculture urbaine engendre une réelle et prégnante attraction de la part d'une multiplicité d'acteurs, des politiques aux citoyens, des architectes urbanistes paysagistes aux promoteurs immobiliers ou encore des chercheurs aux entrepreneurs, pour n'en citer que quelques-uns.

2 Les premiers jardins collectifs, initialement appelés jardins ouvriers, furent créés à la fois par des personnalités remarquables, industriels ou médecin, telles que le docteur Lancry à Dunkerque ou Félicie Hervieu à Sedan et l'abbé Lemire, fondateur des jardins ouvriers et de leur association de tutelle, «la Ligue du foyer et de la terre » (LFCT). La question du foncier et de la disponibilité de terrains cultivables est centrale, depuis toujours, pour la sauvegarde des jardins. Le foncier urbain est et sera une ressource rare et enviée. Aujourd'hui, ce sont les collectivités et les citadins qui impulsent la 
création et l'implantation de jardins collectifs. Pour cela, les municipalités adoptent depuis peu des programmes et modifient leurs plans d'urbanisme pour laisser la place à ces jardins ${ }^{3}$.

3 Bordeaux Métropole ne fait pas exception au développement des jardins collectifs ; cependant, aucune recherche récente, notamment sous l'aspect économique, n'a été conduite sur ce territoire. Ainsi, notre étude et notre propos ont pour objectif à la fois de recenser les diverses formes de jardins collectifs et de mettre en perspective leur multifonctionnalité4. En effet, la littérature fait état des nombreux bénéfices apportés par les jardins et le jardinage, surtout lorsqu'il est collectif. Nous nous proposons donc d'organiser notre propos autour de deux grandes parties : la première est consacrée à l'histoire et à l'évolution des jardins collectifs à Bordeaux et sa métropole à partir, en particulier, d'une analyse bibliographique des comptes rendus des congrès de la LFCT ; la deuxième partie concerne plus spécialement l'analyse de la typologie et des fonctionnalités de cinq jardins collectifs, à partir d'une enquête qualitative et quantitative. Cette partie de la recherche expose le fonctionnement et les externalités positives des jardins collectifs au regard de la fonction alimentaire.

\section{Les jardins collectifs de Bordeaux du XIX siècle à nos jours}

\section{Bordeaux, d'une action sociale volontariste à l'intégration sociale}

4 Durant les premières années $\mathrm{du} \mathrm{xx}^{\mathrm{e}}$ siècle, Bordeaux et sa banlieue n'échappent pas à l'enthousiasme pour les jardins ouvriers, connus sous le vocable de «jardins de l'abbé Lemire ». Le contexte idéologique et industriel bordelais est différent de celui du nord de la France, territoire de naissance des jardins ouvriers. L'habitat ouvrier bordelais est, majoritairement, de type échoppe. L'échoppe est une maison individuelle basse et de plain-pied étroite et profonde, avec un jardin allongé situé à l'arrière de l'immeuble. Dissociés des habitations, les premiers jardins ouvriers bordelais apparaissent, en 1905, sous l'impulsion de Charles Cazalet (1858-1933), maire de Bordeaux. Il fonde l'Cuvre bordelaise des jardins ouvriers avec la première installation de deux groupes de jardins dans le quartier de la Bastide, rive droite. Un troisième groupe voit le jour en 1907 dans le quartier de Saint-Augustin, comprenant 22 parcelles, réparties entre autant de familles nombreuses, critère d'attribution d'un jardin.

Les notables bordelais vantent les bienfaits de ces jardins ; le commandant Grandjean déclare qu'« en rendant service à d'honnêtes et laborieux ouvriers, en cherchant à leur procurer plus de bien-être, nous travaillons en même temps à augmenter la population de notre pays ». Charles Cazalet, dans son rapport au congrès de 1906, relate que le jardin ouvrier est le mode de secours qui coûte le moins cher car le produit récolté représente quatre à six fois la dépense initiale. Il rappelle, en 1910, que les jardins ouvriers sont classés en 1905 " dans l'armement antituberculeux ». À cette époque, d'un point de vue moral, le travail de la terre a sur le travail industriel le grand avantage de favoriser l'épargne et d'enseigner l'économie. En effet, le produit de son travail appartient à l'ouvrier. "L'ouvrier y va le dimanche avec la marmaille, on pioche, on bêche la salade, on y mange au grand air et le soir on rentre fatigué $»^{6}$.

En 1907, six séries de jardinets existent dans les quartiers nord de Bordeaux, proches des industries navales. En 1909, l'œeuvre des jardins ouvriers de Bordeaux revendique 
105 jardins qui profitent à 479 enfants. En 1912, sont dénombrés 40 groupes de jardins et 188 jardins annexés à la Société bordelaise des HBM, pour une superficie totale de 4,70 ha. Après 1914, le nombre de jardins est porté à 197 pour 908 enfants, sous l'impulsion de la famille de Luze, négociants viticoles qui se révèlent les plus fervents défenseurs des jardins ouvriers. En 1923, 417 jardins, de $250 \mathrm{~m}^{2}$ de moyenne, représentent une superficie totale de 12 ha. Le règlement de la Société des jardins de Bordeaux et banlieue, émanation de la LCTF, énonce ses exigences en matière de propreté des jardins, de bonne conduite, de prêts d'outils, de semences.

7 Après la Première Guerre mondiale, un premier déclin atteint à la fois la construction de logements $\mathrm{HBM}^{7}$ et les jardins ouvriers. En 1938, on compte à Bordeaux seulement 60 jardins ouvriers officiels pour deux groupes, cours de Luze et boulevard AlfredDaney, alors que dans le même temps, plus de 30000 jardins individuels sont recensés. Durant, la Seconde Guerre mondiale, nouveau regain des jardins portés essentiellement par des industriels qui installent, en 1941, 760 jardins organisés en 20 groupes adhérant à la Société des jardins ouvriers de Bordeaux et sa banlieue; en 1943, on dénombre 33 groupes et 1433 jardins.

8 À partir de 1946 survient une nouvelle désaffection de ce mode d'action sociale, à la connotation pétainiste et paternaliste, et témoin des amères privations de la guerre, de l'archaïsme économique à l'heure de l'industrialisation agricole. De plus, lors de l'urbanisation intense, orchestrée par une volonté de modernisme, les jardins ouvriers sont jugés laids et désordonnés. Il ne reste plus, en 1950, que 15 groupes et 825 jardiniers officiels. Bien que les jardins soient devenus «familiaux " par la loi de 1952, en lieu et place d'«ouvriers", la pression foncière due, d'une part, à l'augmentation de l'habitat et, d'autre part à l'installation de grandes entreprises telles Michelin ou des raffineries le long du fleuve, font qu'en 1961 seuls perdurent six groupes et 600 jardins. Ces jardins situés dans les zones périurbaines disparaissent petit à petit: en 1965, la Société ne gère plus que cinq groupes dont quatre nouveaux pour 97 jardins 8 .

9 Après le choc pétrolier (1973), une troisième période d'intérêt pour les jardins se profile, provoquée par la réapparition d'une pauvreté de masse associée aux phénomènes d'exclusion et de chômage en constante progression. Dans les années 1980, des municipalités, des associations nationales, des entreprises créent dans les banlieues bordelaises des jardins «familiaux » reprenant les principes généraux de la Ligue du coin de terre et du foyer (LCTF), devenue en 1992 la Fédération nationale des jardins familiaux (FNJF). Ainsi, à Gradignan, 30 parcelles de $200 \mathrm{~m}^{2}$ sont créées sous l'impulsion du maire, ou encore, rive droite, à Cenon et Floirac, naissent respectivement 51 parcelles de $220 \mathrm{~m}^{2}$ et 44 parcelles de $150 \mathrm{~m}^{2}$ sur des terrains appartenant à la SNCF et gérés par l'association Jardinot ${ }^{9}$. Les comités locaux de ces associations gèrent les jardins en attribuant les parcelles (aux plus démunis, à ceux qui habitent en appartement, dans le quartier, etc.), en collectant les cotisations, en assurant les approvisionnements en eau, en graines et en outils.

Un autre type de jardin familial, avec une réalité sociale bien différente, fait son apparition à la fin des années 1990. Il s'agit là de répondre à la crise de l'intégration sociale et culturelle en amenant, par le jardinage, des populations frappées d'exclusion sociale à consolider leur vie privée et à s'initier à la vie publique. Ainsi, les modalités opérationnelles mises en œuvre sont-elles différentes de celles des jardins ouvriers du $\mathrm{XIX}^{\mathrm{e}}$ siècle. L'expérience et la création des jardins familiaux de la cité des Aubiers, à 
Bordeaux, est exemplaire en ce sens. C'est un quartier d'habitat social constitué de tours, avec une population multiethnique d'une grande précarité, comportant un taux élevé de chômeurs et de parents isolés. L'association "Les Jardins d'Aujourd'hui ${ }^{10}$ ", après un long chemin de concertation avec les habitants et de nombreux acteurs sociaux, convainc la municipalité d'installer une cinquantaine de parcelles au pied des immeubles. Grâce à ce nouveau savoir-faire d'animation dans le développement social ${ }^{11}$, le fonctionnement des jardins a été, jusqu'à la disparition de l'association, une réussite en termes d'économie, d'apport d'une meilleure qualité alimentaire pour les jardiniers, de sociabilité et d'ouverture entre communautés. En effet, elles se côtoient, échangent des savoir-faire, s'entraident. Les jeunes sont initiés de manière sensible à la valeur du travail ainsi qu'à une alimentation équilibrée. Ces jardins de développement social nécessitent non seulement un diagnostic de la spécificité du territoire mais aussi une connaissance de la culture et du mode de vie des bénéficiaires, ainsi qu'une animation professionnelle pour dynamiser la vie collective de populations complexes. Ces actions peinent malgré tout à toucher et mobiliser une part non négligeable de personnes en très grande précarité ou exclues.

11 Jusqu'à la fin du $x^{e}$ siècle les jardins familiaux jouent ainsi, d'une part, le rôle d'action sociale dans les quartiers concernés par les politiques de la ville et, d'autre part, servent d'espaces de loisir à une partie de la population, nostalgique de la nature et des savoir-faire agricoles. Le xxI siècle voit apparaître d'autres fonctions et externalités positives des jardins collectifs, que nous allons aborder.

\section{Le jardin partagé, nouveau jardin collectif}

12 Dans les années 2000 se manifeste une nouvelle dynamique d'implantation de jardins collectifs productifs (légumes, fleurs) dans Bordeaux intra-muros et dans quelques communes formant la communauté urbaine de Bordeaux ${ }^{12}$ (CUB) qui devient en 2014 Bordeaux Métropole. La création des jardins collectifs n'est plus majoritairement portée par des industriels ou des associations nationales telles Jardinot ou la FNJF mais par les municipalités, sous la pression de collectifs d'habitants. Les jardins familiaux ne sont plus, en nombre, le type de jardins le plus créés. Leur sont préférés les jardins dits partagés, installés dans les interstices ou les dents creuses des villes, au pied des immeubles ou sur l'espace public. Ainsi, à l'heure actuelle, à Bordeaux et Bordeaux Métropole coexistent deux types de jardins collectifs : les jardins partagés et les jardins familiaux.

Le "jardin partagé», nouveau vocable, est constitué de parcelles de petite taille, cultivées par un ensemble d'urbains; la règle, c'est le collectif. Ces jardins se font sous l'impulsion d'associations telles que les "Incroyables Comestibles ${ }^{13}$ » ou d'associations de quartier ou de collectifs d'urbains défendant une alimentation plus saine et naturelle, une consommation de produits locaux, la sauvegarde de semences paysannes et de variétés anciennes. Ces jardins sont souvent installés sans autorisation, puis, petit à petit, la municipalité met en place une politique pour étayer leur réussite et leur organisation. Elle accompagne en autorisant les différents collectifs à occuper temporairement des micro-parcelles ou espaces publics. Dans l'espace privatif, de nombreuses réalisations éclosent, notamment au pied des immeubles, non seulement dans l'habitat social mais aussi dans des résidences privées, à l'initiative d'habitants 
soucieux d'améliorer leur cadre de vie en transformant les espaces verts en paysages comestibles.

14 Les premiers jardins partagés apparaissent à Bordeaux, puis l'ensemble des 28 communes de la Métropole ${ }^{14}$ se mobilise et s'implique dans cet essor. La progression des jardins partagés n'est pas la même au sein des 28 communes, pour des raisons à la fois liées à la densité et à la diversité de la population, à la typologie de l'habitat et à la présence d'espaces agricoles (forêt ou plaines). D'une part, plus l'on s'éloigne du centre bordelais, plus l'habitat est constitué de lotissements de maisons individuelles possédant leur espace de jardin. Cependant, ces dernières années, toutes les communes connaissent une profonde densification de leur population sous la forme de nouveaux quartiers composés essentiellement d'immeubles de plusieurs étages. D'autre part, certaines communes, principalement situées dans le quart nord-est de Bordeaux, rives gauche et droite de la Garonne, telles Le Haillan, Bruges, Ambès, conservent une tradition de production agricole, qu'elle soit maraichère, d'élevage ou viticole ${ }^{15}$. Ceci permet aux habitants de s'approvisionner localement en légumes frais, sur les marchés notamment, ou de cultiver leur propre potager.

En 2017, une trentaine de jardins partagés à Bordeaux intra-muros est recensée, représentant 6 ha pour 250776 habitants. Deux jardins ouvriers dans les quartiers de Bacalan (24 parcelles de $100-150 \mathrm{~m}^{2}$ sur une parcelle de $3000 \mathrm{~m}^{2}$ ) et des Aubiers (70 parcelles représentant environ $9500 \mathrm{~m}^{2}$ ). Au sein de Bordeaux Métropole, le nombre de jardins partagés et familiaux n'est pas connu précisément (environ 150 jardins partagés en décembre $2017^{16}$ et seulement cinq jardins familiaux). Au sein de Bordeaux Métropole, les jardins partagés représentent la forme prédominante de création de jardins collectifs.

Les règles d'attribution et de fonctionnement et l'accompagnement des jardins partagés relèvent des municipalités et du service "espaces verts" de Bordeaux Métropole. Plusieurs conditions sont nécessaires pour la mise à disposition d'espaces collectifs: la constitution d'une association par les jardiniers, la signature d'une convention pour l'occupation de l'espace, l'existence d'une mixité sociale et l'implication d'écoles et d'associations de quartier ou d'insertion. "Le jardin partagé est fait pour tous, pas besoin de savoir jardiner; le jardinage s'apprend par l'échange avec des jardiniers plus expérimentés, cela permet de tisser des liens ", selon les propos d'un agent territorial. La mixité sociale est demandée afin que ces espaces publics productifs ne soient pas uniquement réservés à une population plus initiée ou plus aisée.

17 Beaucoup de demandes de jardins proviennent actuellement de " nouveaux » jardiniers urbains, le plus souvent néophytes ou peu expérimentés. Le manque d'expérience implique un suivi de la part de la collectivité. Le bon fonctionnement pérenne du jardin et du groupe très hétérogène, dans le temps, nécessite des compétences d'animation et maraîchères. Pour donner les meilleures chances de réussite et de durabilité à ces jardins partagés Bordeaux Métropole sélectionne, sur son territoire, des structures le plus souvent associatives ou d'économie sociale et solidaire qui accompagnent la création du jardin, sa gouvernance et son fonctionnement. Trois partenaires œuvrent avec la Métropole pour la création de nouveaux jardins collectifs. Ces trois organisations, Place au jardin, Le Bocal local, et Les Possibilistes, ont une charte d'intervention définie par la métropole: «ils accompagnent les acteurs (habitants, Villes, bailleurs sociaux) pendant deux ans dans les différentes étapes de la création de 
leur jardin: diagnostic, mobilisation des habitants, choix des usages, règles de fonctionnement, mise en place, animations et chantiers participatifs ». Ces organisations possèdent des compétences maraîchères et d'animation ou de capacité à travailler avec des publics diversifiés et parfois éloignés de l'emploi ou d'une alimentation saine et de qualité.

Chaque jardin partagé constitue une association qui contracte avec la Ville une convention de mise à disposition du terrain; il revient toutefois aux membres de décider du fonctionnement. La gouvernance de l'association peut prendre la forme d'une gouvernance collégiale ou, plus classique, avec une présidence et un bureau. Bordeaux Métropole apporte des conseils techniques et une aide financière pour l'installation du jardin, pouvant aller jusqu'à $80 \mathrm{k€}$. Le respect de la biodiversité, de l'environnement et l'interdiction de l'usage des pesticides y sont prônés à la fois par les jardiniers et les collectivités. L'ensemble des pratiques maraîchères existe, de la culture en pleine terre ou hors-sol, si le sol est pollué, au pied des immeubles, tout en collectif ou tout en parcelles individuelles, ou un mélange de parcelles individuelles et collectives.

\section{Cinq jardins collectifs dans et hors Bordeaux Métropole}

\section{Jardins collectifs, une variété de typologies}

Nous avons étudié cinq jardins collectifs : quatre situés sur les communes de Bordeaux, Cenon, et Gradignan, appartenant à Bordeaux Métropole, et un situé à Canéjan, commune limitrophe de la métropole. Les deux typologies, jardin familial et jardin partagé, sont présentées (Tableau 1). Les jardins familiaux des Aubiers ont été créés en 1993, ceux de l'Arriga à Canéjan en 2016, ceux du Cheminot à Cenon en 1977 et ceux de Gradignan en 1982. Ils sont élaborés selon les principes des jardins ouvriers de l'abbé Lemire, avec des parcelles individuelles. Cependant, leur origine et leur gouvernance sont différentes. Le jardin du Parc Rivière est dit jardin partagé faisant exception à la règle car il reprend la division en parcelles individuelles pour des raisons historiques, et c'est ce qui fonctionne le mieux. Situé en cœur de ville, ses parcelles sont très petites, entre 6 et $12 \mathrm{~m}^{2}$.

Le jardin de Cenon, créé à l'initiative de la SNCF, qui souhaitait "donner à son personnel l'occasion de garnir les assiettes des familles modestes de légumes frais ", est implanté sur un terrain de forme très allongée, le long d'une voie ferrée, (environ $400 \mathrm{~m}$ sur quelques dizaines de mètres de large). La taille des 51 parcelles est comprise entre 50 et $220 \mathrm{~m}^{2}$. Les abris et locaux des outils sont individuels, regroupés par huit dans une cabane de forme polygonale avec un auvent servant d'abri les jours de pluie ou de soleil très intense. La centralité des huit permet échanges ou moments conviviaux de partage. Ici, les jardiniers, hommes et femmes, sont presque exclusivement des retraités avec de faibles revenus. Ce jardin dépend de l'association nationale "Jardinot", qui sert aussi de centrale d'achat pour les semences et les produits de protection des cultures utilisables en agriculture bio. Jardinot publie une revue mensuelle dont le prix est compris dans la cotisation annuelle, de $53 €$ par $100 \mathrm{~m}^{2}$, et dispense des formations pour l'apprentissage de pratiques plus respectueuses de l'environnement. Une labellisation des jardins par des «Jardinot de 
bronze, d'argent ou d'or " permet aux jardins d'évaluer leur progression dans leurs démarches de protection de l'environnement.

21 Le jardin des Aubiers comporte 70 parcelles de 100 à $200 \mathrm{~m}^{2}$. Les productions sont variées, suivant le besoin de la famille ou le pays d'origine des jardiniers. Ici, les cabanes sont des petits abris individuels recouverts de tôle ondulée, les parcelles individuelles étant organisées autour de plusieurs abris. Les jardiniers des Aubiers sont très accompagnés et soutenus par Bordeaux Métropole sous la forme de la mise à disposition d'un animateur-jardinier à temps plein. Il a la charge de faire respecter les règles, de régler les conflits entre jardiniers, de gérer avec eux l'entretien des réseaux d'eau et des cabanes. En effet, il arrive que des conflits éclatent, ou que les règles de propreté (emballages ou dépôts jetés et laissés) ne soient pas respectées. Les réseaux d'eau sont anciens, régulièrement sujets aux fuites. Le comptage de la consommation d'eau est individuel et son règlement annuel fait l'objet de discussions, voire de contentieux quand le montant est jugé trop élevé. Deux réunions par an sont organisées par l'animateur afin d'aborder avec l'ensemble des points de gestion du jardin : entretien, eau, cotisation, nouveaux arrivants, etc.

22 Trente parcelles, majoritairement de $200 \mathrm{~m}^{2}$, composent les jardins de Gradignan, réalisés sous l'impulsion conjointe de la mairie et d'un collectif d'habitants ne possédant pas de jardin individuel. Les parcelles et leurs cabanes sont organisées de façon originale, par groupe de six autour d'un point d'eau commun de façon à optimiser l'espace et les réseaux. Les jardiniers sont ici majoritairement des retraités dont le jardinage est le loisir. Avec l'arrivée de nouveaux jardiniers (famille monoparentale ou jardiniers avec emploi), quelques parcelles ont été partagées pour créer des parcelles de $50 \mathrm{~m}^{2}$. Depuis leur origine, les jardins familiaux se doivent d'être bien entretenus et tout particulièrement, les parties communes. Ainsi, à Gradignan, chaque année, deux ou trois journées de travaux collectifs permettent d'entretenir les allées, de restaurer des outils ou des jardinières ou encore de cultiver des parcelles utilisées pour les ateliers scolaires ou thérapeutiques. Une mare a aussi été implantée à la fois pour encourager à la fois la biodiversité et à des fins éducatives. Sous forme associative, les jardins de Gradignan sont membres de la FNJF et en sont le représentant local. Les jardiniers tiennent à cette affiliation car la FNJF est l'un des interlocuteurs privilégiés des ministères, assurant la défense des jardins et permettant au niveau national des échanges de savoir-faire sur de nouvelles pratiques, notamment pour une meilleure protection de l'environnement ou sur les questions de sécurité alimentaire.

Quant au jardin de Canéjan, né en 2016 de la volonté politique de la municipalité qui l'a inscrit dans son Agenda $21^{17}$, il est construit en concertation avec des personnalités qualifiées de la société civile, les conseils des sages et des jeunes et des associations. Ce projet est très fortement porté par les élus, et lors des dernières élections municipales, il fut le sujet de fortes discussions. "Le maire souhaite concilier par les jardins familiaux l'intérêt économique par la production de légumes et fruits sains assurant un complément alimentaire aux jardiniers, l'équité sociale par la création de liens sociaux, culturels, intergénérationnels et promouvoir l'échange de savoirs, la solidarité et la fonction pédagogique en favorisant l'éducation des enfants à l'environnement et à l'alimentation et la protection de l'environnement par la pratique d'un jardinage utilisant produits et méthodes naturels ». La surface totale occupée par les 61 jardins et les différentes infrastructures est de $4355 \mathrm{~m}^{2}$, entre une forêt de pins et un lotissement. Elle se décompose en 21 parcelles de $20 \mathrm{~m}^{2}, 26$ parcelles de $50 \mathrm{~m}^{2}, 11$ parcelles de $80 \mathrm{~m}^{2}$, 
une parcelle pour les écoles $\left(100 \mathrm{~m}^{2}\right)$, une parcelle pour le $\operatorname{CCAS}^{18}\left(100 \mathrm{~m}^{2}\right)$ et une parcelle pour le conseil municipal des jeunes $\left(100 \mathrm{~m}^{2}\right)$. Chaque parcelle est clôturée et possède sa cabane. Ce sont, ici aussi, les jardiniers qui organisent et réalisent des ateliers pour les écoliers.

Le jardin partagé du Parc Rivière, situé au cœur de Bordeaux, est composé de petites parcelles individuelles de 6 à $9 \mathrm{~m}^{2}$ ne permettant pas une autonomie alimentaire. Aujourd'hui, constitué en association, ce jardin accueille des personnes d'origines très diverses tant géographiques que culturelles ou sociales. Des parcelles communes sont cultivées par des écoles ou des associations de quartier ou l'association ERASMUS des étudiants étrangers qui aiment le jardinage et font partager les us et coutumes ou variétés de leur pays d'origine. La petitesse des parcelles fait que les jardiniers débordent d'inventivité afin d'avoir une production optimale et originale pour que la parcelle soit belle. L'attribution des parcelles se fait sur demande, en fonction de critères géographiques (il faut habiter dans le quartier ou dans la commune), et/ou sociaux (revenus, famille monoparentale). Les jardiniers attendent parfois plusieurs années pour obtenir une parcelle. En janvier 2018, le jardin forme une association, selon les vœux de la municipalité, de gouvernance collégiale.

Depuis le XIX ${ }^{e}$ siècle, les modes de vie ont évolué. Les familles sont plus petites et disposent de moins de temps. Les jardins familiaux sont divisés pour offrir des superficies réduites allant de $50 \mathrm{~m}^{2}$ à $100 \mathrm{~m}^{2}$ plutôt que de 200 à $450 \mathrm{~m}^{2}$. La fourniture d'eau, essentielle à la culture maraîchère, est assurée soit par un puits soit par la ville, toutefois, la récupération des eaux de pluie est non seulement recommandée mais toujours pratiquée. En cœur de ville, les jardins partagés avec des parcelles très petites se développent dans des interstices ou «dents creuses» des villes avec une gouvernance associative. De plus, la typologie des jardiniers évolue: même si les retraités sont encore majoritaires, de plus en plus de jeunes et de femmes seules demandent des jardins. Les crises économique et sanitaire étant passées par là, comme motivation, le loisir fait souvent place à la recherche d'une meilleure alimentation.

\section{Multifonctionnalité versus alimentation dans les jardins collectifs ?}

Les jardins collectifs, une des formes d'agriculture urbaine, possèdent des fonctions, regroupées sous le concept de multifonctionnalité ${ }^{19}$, qui évoluent. On reconnaît à l'agriculture urbaine des fonctions additionnelles à la fonction productive alimentaire. Concernant les jardins collectifs, qu'ils soient familiaux ou partagés, la littérature indique des externalités et impacts positifs relevant de bénéfices sociaux comme la création de liens, de bénéfices dans le "réapprendre à manger", d'une meilleure production alimentaire, voire l'autonomisation, d'un développement économique par l'insertion de populations éloignées de l'emploi ou en termes de santé humaine. La nature en ville est un élément majeur de la santé physique et mentale des humains tant par un contact tactile que visuel ${ }^{20}$. Selon Éric Duchemin ${ }^{21}$, les fonctions d'" aménagement urbain», de "développement économique », de "loisir», de "création de lien social», de "sécurité alimentaire», de "préservation de l'environnement », d'« éducation ", portées par les formes d'agriculture urbaine, ont des degrés d'importance très variés selon le type de jardin, la localisation, le jardinier, l'appartenance à un collectif ou à une institution publique de la personne qui en parle. 
Notre enquête qualitative cherche à évaluer les bénéfices que les jardiniers tirent du jardin, notamment en termes de production et d'une certaine autonomie alimentaire. Des entretiens semi-directifs ont été menés auprès de dix-huit jardiniers des cinq jardins et quatre acteurs publics, à savoir la chargée des jardins collectifs de Bordeaux Métropole, un élu de la mairie de Bordeaux, la chargée de communication de la mairie de Canéjan et le maire de Gradignan. De façon simultanée, une enquête quantitative a été réalisée auprès de quinze jardiniers volontaires appartenant à trois des jardins étudiés.

L'ensemble des cinq jardins représente 261 jardiniers qui souhaitent jardiner non seulement pour leur loisir, comme ils nous l'ont déclaré, mais aussi pour produire quelques légumes et petits fruits de bonne qualité gustative et sains. Un jardinier relate : «On vient ici pour le partage, pour le plaisir, pour échanger sur notre passion, prendre l'air et avoir quelques légumes de bonne qualité, on ne fait pas forcément d'économies. » En effet, le prix de «location » n'est pas très élevé, voire symbolique, mais «le coût des semences et des plants représente une partie importante dans le budget, et on ne compte pas le temps passé » nous exprime la majorité des jardiniers. La convivialité et l'entraide sont la règle dans l'ensemble de ces jardins, des piqueniques sont organisés ainsi que des journées de travail collectif pour nettoyer ou réparer locaux ou outils communs. De plus, les jardiniers d'un même jardin se connaissent tous entre eux, quand l'un est absent, son voisin entretient sa parcelle et l'arrose, par exemple.

Le jardin est créateur de lien social : "C'est l'occasion de se retrouver ensemble et de partager savoir-faire, connaissances sur les meilleures semences ou variétés, par exemple, etc. ", disent les jardiniers interviewés. Des jardiniers retraités, âgés et seuls nous disent qu'ils «ont dans les jardins des amis avec lesquels discuter et ils ont l'impression d'être encore utiles à la société en transmettant leurs pratiques ». Des semences et des légumes sont échangés : "Quand les salades arrivent toutes en même temps, j'en donne à mon voisin qui n'en plante pas » nous explique une jardinière. Le jardin est aussi lieu d'insertion sociale; en effet, pratiquer la même activité permet à des personnes d'origine et de langue différentes d'apprendre à se connaître et de s'intégrer.

En termes d'environnement, les jardins sont des poumons verts au cœur des villes dont la température est élevée en été. Les jardiniers nous expliquent qu'ils y viennent "se rafraîchir » en soirée ou le matin tôt : "La température est plus supportable que dans [leur] appartement.» Les pratiques respectueuses de l'environnement pour le maraîchage comme le paillage, le compostage ou l'utilisation de plantes compagnes pour éviter certaines maladies sont recommandées et appliquées.

31 En termes de fonction sanitaire, des parcelles communes, présentes dans trois jardins, sont exigées par les municipalités afin de favoriser les fonctions éducative et thérapeutique du jardinage. Elles sont destinées à des associations ou des jardiniers et cultivées par eux. Des ateliers de jardinage ou de cuisine collective à l'intention des enfants ou des adultes éloignés de l'emploi ou des patients d'hôpitaux psychiatriques sont organisés. Cela permet d'apprendre la saisonnalité des productions mais aussi de réapprendre à cuisiner de façon variée et à manger sain afin de prévenir l'obésité ou le diabète. En échange de ce service, les collectivités accordent une subvention aux jardins. 

jardiniers qui sont interrogés ici. En effet, ils ne quantifient jamais, ni en poids ni en économies, leurs récoltes. Toutefois, ils disent « pouvoir ainsi manger de vrais légumes, plus sains et meilleurs au goût ». Beaucoup de jardiniers affirment : «Pendant la saison, on achète moins de légumes, mais on ne peut tout produire ", un autre nous confiera : «Pendant la saison, de mai à septembre, nous n'achetons pas de légumes et j'en donne à mes enfants, mais je ne crois pas que l'on fasse des économies, les produits sont meilleurs, je n'ai pas les moyens d'acheter du bio tous les jours. » comptabiliser leur production au moyen d'une enquête sur la base du volontariat auprès des trois jardins familiaux de Cenon, l'Arriga et Gradignan. L'enquête a été réalisée avec un carnet de récolte simple, fourni aux jardiniers volontaires, à charge pour eux de peser leurs récoltes entre le mois de mai et fin août 2017 et d'inscrire, par type de produits, la date de la récolte ainsi que son poids. Quinze jardiniers ont accepté de mener cette expérience sur les 137 sollicités. Ils ont cependant tous dit : "Mais ce n'est pas facile, je ne sais pas si je vais y arriver et pouvoir m'y tenir. "

Les carnets de récolte ont été distribués entre le 29 mai et le 10 juin 2017 et récupérés entre le 4 septembre et le 20 septembre 2017. Les résultats (Tableau 2) montrent que les indications des périodes de récoltes sont variables d'un jardin à l'autre et entre jardiniers d'un même jardin. Cela est dû non seulement aux variations de dates de plantation et du temps de pousse des fruits et légumes mais aussi au caractère contraignant de cette enquête. "Je n'ai pas noté exactement les dates, ni le poids, j'ai estimé ", disent plusieurs d'entre eux. De plus, pour le jardin de Gradignan, les dates indiquées, ainsi que les poids, ont réellement fait l'objet d'une estimation, à partir du nombre de plants cultivés par espèce. En effet, les jardiniers n'ont pas été assez motivés et ont oublié de remplir le carnet !

les types de légumes produits, le tableau 3 montre que les tomates, les herbes aromatiques et salades sont produites par $100 \%$ des jardiniers répondants, les courgettes et les pommes de terre par $87 \%$, les haricots par $74 \%$, les poivrons et piments par $53 \%$, les oignons, poireaux, aubergines et choux par $40 \%$, les betteraves et concombres par $33 \%$, les carottes et artichauts par $27 \%$ puis en dernier, les petits pois, avec $13 \%$. Les topinambours ne sont cultivés que par un seul jardinier. Quant aux petits fruits, les fraises sont cultivées par $60 \%$ des jardiniers, les framboises par $47 \%$, le cassis et la rhubarbe par deux jardiniers dont un qui produit $40 \mathrm{~kg}$ de cassis par an, ce qui est rare et notable.

Pour déterminer les quantités produites par $\mathrm{m}^{2}$, on a additionné les poids de l'ensemble des légumes et petits fruits déclarés, excepté les salades, les concombres et cucurbitacées, comptés par unité et pas pris en compte ici. Rappelons que les quantités indiquées par les jardiniers de Gradignan sont évaluées par eux-mêmes et ne correspondent pas à des pesées effectuées. Le tableau 2 montre une grande disparité dans les quantités produites par $\mathrm{m}^{2}$, avec des valeurs extrêmes comprises entre $0,16 \mathrm{~kg} /$ $\mathrm{m}^{2}$ et $3,02 \mathrm{~kg} / \mathrm{m}^{2}$. Elles ne semblent pas être en relation avec le jardin (la qualité du sol ou l'exposition) ni avec le genre du jardinier. Le jardinier J1 du jardin familial de Cenon qui a produit $0,16 \mathrm{~kg} / \mathrm{m}^{2}$ explique que sa faible productivité est due à la maladie, qui l'a empêché de planter ses légumes et petits fruits à temps, et aux intempéries, sa parcelle ayant été inondée pendant de nombreuses semaines. Avec un certain fatalisme, il dit: 
«Cette année, les tomates n'ont pas donné, j'ai raté les fraises, même les salades n'ont pas aimé, je ne sais pas pourquoi, cette année j'en ai raté beaucoup ».

37 Les disparités de productions peuvent aussi s'expliquer par le temps consacré aux jardins, plus ou moins important, la densité des légumes plantés par $\mathrm{m}^{2}$ ainsi que les variétés de légumes, facteurs absents de notre enquête. Selon Le Guide Terre vivante du potager $b_{i o^{22}}$, le rendement des pommes de terre est compris entre 15 et $60 \mathrm{~kg} / 10 \mathrm{~m}^{2}$, celui des tomates entre 50 et $100 \mathrm{~kg} / 10 \mathrm{~m}^{2}$ et les poireaux $25-50 \mathrm{~kg} / 10 \mathrm{~m}^{2}$. Pour la même superficie cultivée, les tomates ont un rendement apparent supérieur par $\mathrm{m}^{2}$ à celui des pommes de terre. Toutefois, les meilleurs rendements résultent d'une attention plus grande à leur production de la part de certains jardiniers. De même, certains ont davantage les "doigts verts " ou réussissent mieux certains légumes, d'autres expriment un impératif de bien faire, de "rentabilité », en ayant produit «plein de légumes pour la famille».

Figure 1

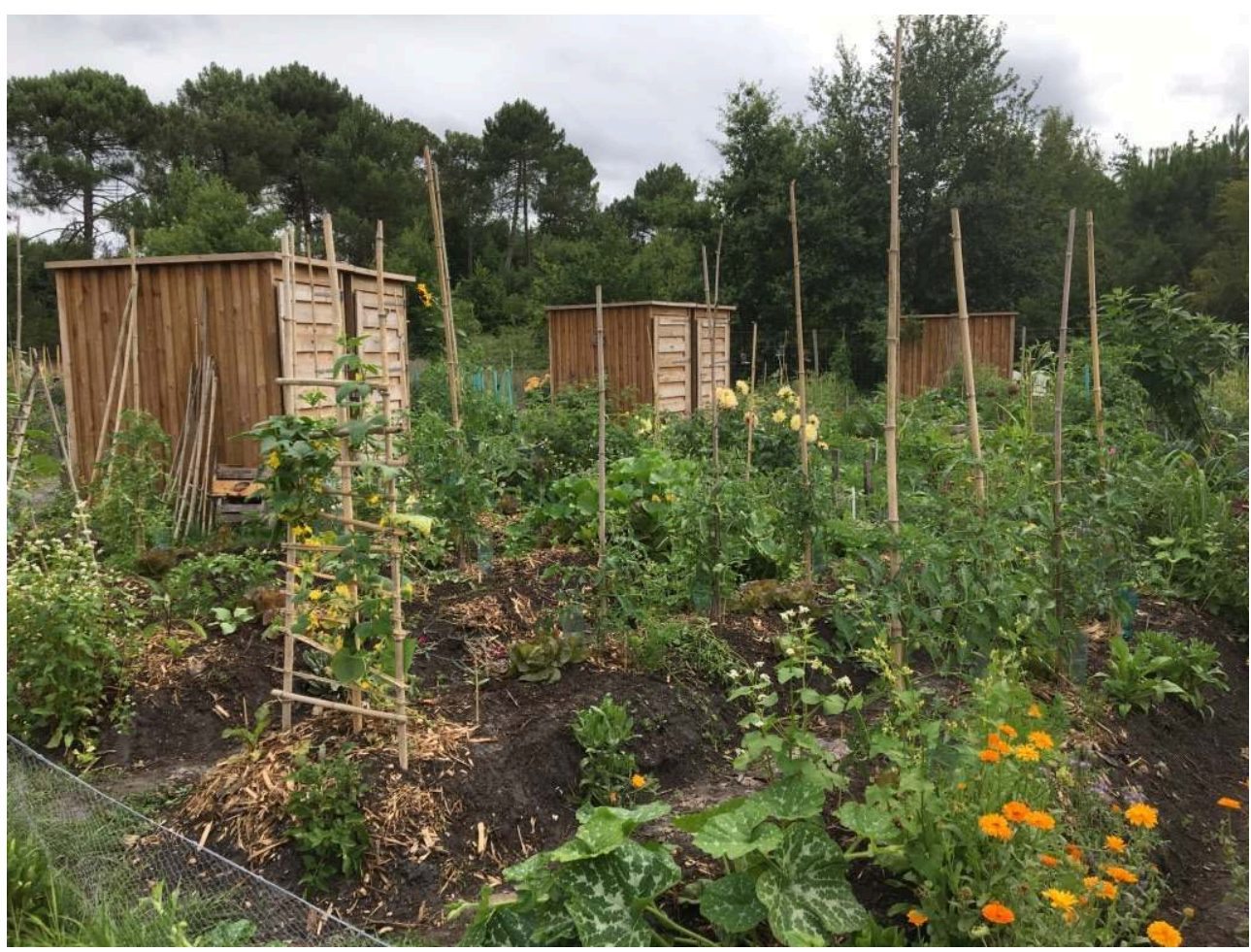

LE JARDIN DE L'ARRIgA À CANÉJAN.

(C) VÉRONIQUE SAINT-GES.

En sus d'une production comestible, les jardins sont fleuris, principalement ceux qui sont confiés à des femmes (fig. 1). Il s'agit de rendre le jardin gai et attractif; une jardinière nous dit qu' « un jardin sans fleurs, ce n'est pas un jardin ». Certaines fleurs sont aussi utilisées comme plantes compagnes pouvant éloigner les maladies, tel l'œillet d'Inde, qui éloigne les pucerons des plants de tomate. Cette étude ne nous permet pas de corréler la production quantitative d'une parcelle et la méthode de culture choisie, traditionnelle, permaculture ou encore agriculture bio. En effet, cela n'a pas été demandé dans les carnets de récolte. Cependant, des modes de culture respectueux de l'environnement et de la biodiversité comme le compostage collectif pour nourrir la terre et favoriser la vie dans les sols et éviter le gaspillage, la 
récupération des eaux de pluie ou encore l'utilisation de semences de légumes anciens, la mise en place d'hôtels à insectes, etc. sont pratiqués.

\section{Conclusion}

39 En conclusion, bien que nos résultats concordent avec la littérature, cette étude a ses limites et elle pourrait être dépassée si l'enquête auprès des jardiniers était conduite de façon plus suivie. Une relève quotidienne, par "l'enquêteur ", des quantités récoltées, des pratiques culturales et des densités des plantations est indispensable pour obtenir des données plus robustes. Peu de jardiniers acceptent de répondre à une enquête, qu'ils ressentent comme une contrainte ou un éventuel jugement négatif si leurs productions sont faibles. La plupart jardinent par plaisir, ne comptent pas leur temps et ne veulent pas d'obligation supplémentaire. Les données fournies et utilisées ici reposent sur la confiance qui leur est accordée.

40 À Bordeaux, la forme de jardin la plus répandue? aujourd'hui est celle des jardins partagés au pied d'immeubles et dans les interstices de la ville qui demandent peu d'espace. La pression du foncier est toujours aussi prégnante, Bordeaux étant une ville actuellement très recherchée par de nouveaux arrivants potentiels. Les jardins et les bénéfices du jardinage en termes de protection de l'environnement, de création de lien social, d'apprentissage d'une meilleure alimentation, d'insertion ne sont pas encore totalement reconnus par tous les acteurs publics. Cependant, la demande forte et pressante des citadins incite à un nouvel aménagement urbain plus vert et une très forte autonomie alimentaire de proximité, même si celle-ci est peu réaliste.

41 En ce qui concerne la fonction alimentaire, « Ce n'est pas la chose que je viens chercher ici » est un constat fréquemment exprimé. Il est difficile de quantifier la réalité de la production, vu le caractère contraignant que revêt la démarche auprès des jardiniers. Cependant, ce qui est important pour tous, « C'est de cultiver et de manger des produits sains avec du goût». Pour la plupart, ils disent ne pas avoir les moyens d'acheter du bio. Quant aux collectivités qui favorisent et accompagnent la création de jardins collectifs, les fonctionnalités qu'elles mettent en avant sont le lien social, la mixité sociale et l'apprentissage d'une meilleure alimentation en lien avec les saisons. Les jardins ont cependant un rôle central dans la vie de la ville, en comparaison avec d'autres activités de loisir, le jardinage permettant une réalisation qui «redonne notamment estime de soi ", comme cela nous a été dit par un agent public.

42 Les jardins collectifs ont des valeurs sociales, au-delà de la fonction productive, tant du point de vue des jardiniers que de certains acteurs sociaux, qu'ils soient publics ou associatifs. Ainsi, lors des entretiens, la majorité des jardiniers trouvent que le jardinage est une "activité créative », qui donne du sens à l'alimentation : «On prend le temps de regarder la nature faire, on reprend conscience des saisonnalités des productions. " De même, les fonctions éducative et créatrice de lien social sont importantes: «Les enfants viennent, on est content de leur montrer comment les légumes poussent », et aussi : "On échange nos méthodes, des plants ou des semences qui produisent bien", ou encore, "Quand on vit seule, c'est agréable de se retrouver pour parler de sa passion, faire des pique-niques, partager de bons moments de rire ».

Tableau 1-1 : Caractéristiques des 5 jardins collectifs 


\begin{tabular}{|l|l|l|l|l|l|}
\hline Localisation & $\begin{array}{l}\text { Nom du } \\
\text { Jardin }\end{array}$ & $\begin{array}{l}\text { Type de } \\
\text { jardin }\end{array}$ & $\begin{array}{l}\text { Nombre de } \\
\text { parcelles }\end{array}$ & $\begin{array}{l}\text { Parcelles } \\
\text { collectives }\end{array}$ & $\begin{array}{l}\text { Taille moyenne des } \\
\text { parcelles }\end{array}$ \\
\hline Bordeaux & Les Aubiers & Familial & 70 & Non & $100-200 \mathrm{~m}^{2}$ \\
\hline Bordeaux & Parc Rivière & Partagé & 54 & Oui & $6-12 \mathrm{~m}^{2}$ \\
\hline Canéjan & De l'Arriga & Familial & 56 & Oui & $20 \mathrm{~m}^{2}-100 \mathrm{~m}^{2}$ \\
\hline Cenon & Du cheminot & Familial & 51 & Non & $50-220 \mathrm{~m}^{2}$ \\
\hline Gradignan & $\begin{array}{l}\text { de } \\
\text { Gradignan }\end{array}$ & Familial & 30 & Oui & $200 \mathrm{~m}^{2}$ \\
\hline
\end{tabular}

Tableau 1-2 : Caractéristiques des 5 jardins collectifs

\begin{tabular}{|l|l|l|l|l|l|l|}
\hline Localisation & $\begin{array}{l}\text { Nom du } \\
\text { Jardin }\end{array}$ & $\begin{array}{l}\text { Type de } \\
\text { jardin }\end{array}$ & $\begin{array}{l}\text { Superficie } \\
\text { totale }\left(\mathrm{m}^{2}\right)\end{array}$ & $\begin{array}{l}\text { Prix } \\
\text { location/ } \\
\text { an }\end{array}$ & Gouvernance & $\begin{array}{l}\text { Fédération } \\
\text { nationale } \\
\text { d'association }\end{array}$ \\
\hline Bordeaux & Les Aubiers & Familial & 9550 & $\begin{array}{l}5 € / \\
\text { parcelle }\end{array}$ & Association & non \\
\hline Bordeaux & $\begin{array}{l}\text { Parc } \\
\text { Rivière }\end{array}$ & Partagé & 600 & $\begin{array}{l}5 € / \\
\text { parcelle }\end{array}$ & Association & non \\
\hline Canéjan & De l'Arriga & Familial & 13000 & $5-10 € / \mathrm{m}^{2}$ & Association & non \\
\hline Cenon & $\begin{array}{l}\text { Du } \\
\text { cheminot }\end{array}$ & Familial & 10000 & $\begin{array}{l}53 € / 100 \\
\mathrm{~m}^{2}\end{array}$ & Association & Jardinot \\
\hline Gradignan & Familial & 7500 & $\begin{array}{l}90 € / 200 \\
\mathrm{~m}^{2}\end{array}$ & Association & FNJF \\
\hline
\end{tabular}

Tableau 2 : Production des jardins familiaux

\begin{tabular}{|c|c|c|c|c|c|c|c|}
\hline \multicolumn{4}{|c|}{ Jardin familial de L'Arriga - Canéjan } & \multicolumn{4}{|c|}{ Jardin familial de Gradignan } \\
\hline Jardinier & Période (2017) & Sexe & $\begin{array}{l}\text { Production } \mathrm{Kg} / \\
\mathrm{m}^{2}\end{array}$ & Jardinier & $\begin{array}{l}\text { Période } \\
(2017)\end{array}$ & Sexe & $\begin{array}{l}\text { Production } \mathrm{Kg} / \\
\mathrm{m}^{2}\end{array}$ \\
\hline $\mathrm{J} 1$ & $\begin{array}{l}12 \text { mai }-4 \\
\text { sept }\end{array}$ & M & 2,3 & $\mathrm{~J} 1$ & mai - 7 sept & M & 0,765 \\
\hline $\mathrm{J} 2$ & 6 juil - 31 août & $\mathrm{F}$ & 1,4 & $\mathrm{~J} 2$ & mai - 7 sept & M & 0,404 \\
\hline $\mathrm{J} 3$ & $\begin{array}{l}13 \text { juil }-31 \\
\text { août }\end{array}$ & $\mathrm{F}$ & 1,5 & J3 & mai - 7 sept & $\mathrm{F}$ & 1,6 \\
\hline
\end{tabular}




\begin{tabular}{|c|c|c|c|c|c|c|c|}
\hline $\mathrm{J} 4$ & $\begin{array}{l}22 \text { juin }-18 \\
\text { sept }\end{array}$ & M & 2,66 & $\mathrm{~J} 4$ & mai - 7 sept & $\mathrm{F}$ & 1,4 \\
\hline J5 & $\begin{array}{l}20 \text { juil }-28 \\
\text { août }\end{array}$ & M & 1,46 & J5 & mai - 7 sept & $M$ & 0,89 \\
\hline J6 & $\begin{array}{l}20 \text { juil }-18 \\
\text { sept }\end{array}$ & M & 3,02 & J6 & mai - 7 sept & $\mathrm{F}$ & 1,3 \\
\hline \multicolumn{4}{|c|}{ Jardin familial de Cenon } & & & & \\
\hline Jardinier & Période & Sexe & $\begin{array}{l}\text { Production } \mathrm{Kg} / \\
\mathrm{m} 2\end{array}$ & & & & \\
\hline $\mathrm{J} 1$ & mai - 12 sept & $F$ & 0,16 & & & & \\
\hline $\mathrm{J} 2$ & $\begin{array}{l}17 \text { juin }-8 \\
\text { août }\end{array}$ & $\mathrm{M}$ & 0,87 & & & & \\
\hline J3 & $\begin{array}{l}11 \text { mai }-7 \\
\text { juillet }\end{array}$ & $\mathrm{F}$ & 0,73 & & & & \\
\hline & & & & & & & \\
\hline & & & & & & & \\
\hline & & & & & & & \\
\hline
\end{tabular}

Tableau 3 : Type de petits fruits et légumes cultivés dans les jardins collectifs

\begin{tabular}{|c|c|c|c|}
\hline $\begin{array}{l}\text { Légumes ou petits } \\
\text { fruits }\end{array}$ & $\begin{array}{l}\text { Nombre de Jardiniers } \\
(\%)\end{array}$ & $\begin{array}{l}\text { Légumes ou petits } \\
\text { fruits }\end{array}$ & $\begin{array}{l}\text { Nombre de Jardiniers } \\
(\%)\end{array}$ \\
\hline Tomates & \multirow{3}{*}{$15(100)$} & Betteraves & \multirow{2}{*}{$5(33)$} \\
\hline Salades & & Concombre & \\
\hline Herbes aromatiques & & Carottes & \multirow{2}{*}{$4(27)$} \\
\hline Courgettes & \multirow{2}{*}{$13(87)$} & Artichaut & \\
\hline Pommes de Terre & & Ail & \multirow{2}{*}{$3(20)$} \\
\hline Haricots & $11(74)$ & Fèves & \\
\hline Fraises & $9(60)$ & Cassis & \multirow{3}{*}{$2(13)$} \\
\hline Poivrons & $8(53)$ & Rhubarbe & \\
\hline Framboises & $7(47)$ & Petit Pois & \\
\hline Oignon & $6(40)$ & Topinambour & \\
\hline
\end{tabular}




\begin{tabular}{|c|c|}
\hline Poireaux & Cacahuète \\
\hline Aubergines & \\
\hline Chou & \\
\hline
\end{tabular}

\section{NOTES}

1. - Ville du Yorkshire (Royaume-Uni) où est né le mouvement « Incredible edible ».

2. - L'agriculture urbaine, selon la FAO (Food and Agriculture Organization), est la culture des plantes et l'élevage des animaux à l'intérieur et aux alentours des villes; voir le site : http:// www.fao.org/urban-agriculture/fr/ [consulté le 05/12/2017].

3. - POURIAS, Jeanne, AUBRY, Christine, DUCHEMIN, Éric. «Is food a motivation for urban gardeners? Multifunctionality and the relative importance of the food function in urban collective gardens of Paris and Montreal ». Agriculture and Human Values, 2016, 33 (2), p. 257-273.

4. - HUANG, Jiao, TICHIT, Muriel, POULOT, Monique, DARLY, Ségolène, LI, Shuangcheng, PETIT, Caroline et AUBRY, Christine. «Comparative review of multifunctionality and ecosystem services in sustainable agriculture ». Journal of environmental management, 2015, 149, p. 138-147.

5. - GRAND, Jean. Visites aux cuvres sociales bordelaises, allocution de M. le commandant Granjean. 1907, p. 25-27.

6. - BROUARDEL, Paul. «Les jardins ouvriers». Dans Compte rendu de la réunion des trois ceuvres bordelaises des HBM, des Bains-douches à bon marché et des Débits de tempérance. Bordeaux: impr.G.Gounouilhou, 1903, p. 24-25. Voir le site: https://gallica.bnf.fr/ark:/12148/ bpt6k5544886t.texteImage [consulté le 03/12/2018].

7. - HBM : Habitation bon marché.

8. - CÉRÉZUELLE, Daniel. Jardins familiaux des Aubiers : bilan d'une expérience bordelaise. PADES/Les Jardins d'aujourd'hui, 1997, p.10-12. Voir le site: https://www.padesautoproduction.net/ [consulté le 03/12/2018].

9. - Jardinot: association anciennement «le jardin du cheminot», voir le site: http:// jardinot.org [consulté le 15/12/2017].

10. - Jardins d'Aujourd'hui : association loi de 1901 créée en 1986, disparue en 2016 ; ses missions d'intérêt général étaient de lutter contre l'exclusion grâce au jardinage collectif et urbain.

11. - CÉRÉZUELLE, Daniel. « Les jardins familiaux, lieux d'initiation à la civilité ». Communications, 2003, 74, «Bienfaisante nature», p. 65-83. Voir le site: https://www.persee.fr/doc/ comm_0588-8018_2003_num_74_1_2129 [consulté le 03/12/2018].

12. - La CUB est composée de 27 communes : Ambarès-et-Lagrave, Ambès, Artigues, Bassens, Bègles, Blanquefort, Bordeaux, Bouliac, Le Bouscat, Bruges, Carbon-Blanc, Cenon, Eysines, Floirac, Gradignan, Le Haillan, Lormont, Mérignac, Parempuyre, Pessac, Saint-Aubin-de-Médoc, Saint-Louis-de-Montferrand, Saint-Médard-en-Jalles, Saint-Vincent-de-Paul, Le Taillan-Médoc, Talence et Villenave d'Ornon.

13. - «Incroyables comestibles» : mouvement citoyen d'agriculture urbaine participative venu d'Angleterre. Ils sont partout et invitent les habitants à planter des légumes en ville partout où 
c'est possible... et à cultiver les liens. Voir le site : http://lesincroyablescomestibles.fr/ [consulté le 05/12/2017].

14. - Voir le site : http://www.bordeaux-metropole.fr [consulté le 05/12/2017].

15. - Selon une enquête réalisée, en 2012, par la chambre d'agriculture de la Gironde, sont répertoriées 176 exploitations agricoles dont 58 viticoles, 47 maraîchères, 33 en élevage, 27 en grandes cultures, 6 horticoles, 2 arboricoles et 3 apicoles (chambre d'agriculture, 2012).

16. - Voir le site : https://rue89bordeaux.com/2017/12/jardiner-courses-cuisiner-alimentationlocale-lien-social/ [consulté le 03/01/2017].

17. - Projet de développement durable.

18. - CCAS : centre communal d'action sociale.

19. - DUCHEMIN, Éric. « Multifonctionnalité de l'agriculture urbaine : perspective de chercheurs et de jardiniers ». Dans DUCHEMIN, Éric (éd.). Agriculture urbaine: aménager et nourrir la ville. Montréal : Vertigo, p. 95-107.

20. - MANUSSET, Sandrine. "Impacts psycho-sociaux des espaces verts dans les espaces urbains ». Développement durable et territoires, 2012, vol.3, $\mathrm{n}^{\circ} 3$. Voir le site: http:// journals.openedition.org/developpementdurable/9389 consulté le 29/10/2017 [consulté le 03/12/2018].

21. - DUCHEMIN, Éric, WEGMULLER, Fabien, LEGAULT, Anne-Marie. «Agriculture urbaine : un outil multidimensionnel pour le développement des quartiers ». Vertigo, 2010, vol. 10, $\mathrm{n}^{\circ} 2$, p. 1-17. Voir le site : https://journals.openedition.org/vertigo/10436 [consulté le 03/12/2018].

22. - THOREZ, Jean-Paul, BOUÉ, Christian. Le Guide Terre vivante du potager bio. Mens: Terre vivante, 2013.

\section{RÉSUMÉS}

Cette recherche présente, dans un premier temps, l'évolution de l'ensemble des jardins collectifs du XIX ${ }^{e}$ siècle à nos jours à Bordeaux et sa métropole. Dans un deuxième temps, leur typologie et leurs fonctions actuelles sont explorées. L'étude montre que les jardins partagés sont les plus implantés mais que les jardins familiaux perdurent dans certaines conditions. Les résultats d'entretiens semi-directifs et une enquête quantitative conduits auprès de cinq jardins collectifs à Bordeaux Métropole attestent que le jardin apporte de nombreux bénéfices autres que la fonction alimentaire. Cette dernière n'est pas prédominante. Les fonctions éducatives, curatives et créatives de lien social sont mises en avant, le plus souvent, par les jardiniers.

This article first of all presents the evolution of collective gardens in Bordeaux and its metropolitan area from the nineteenth century up to the present day. In a second part, there is an examination of their typology and of their current functions. The study shows that shared gardens are the most well-established, but that allotment or family gardens still survive under certain conditions. The results of a programme of interviews and a quantitative survey carried out in five collective gardens in Bordeaux show that these gardens bring many benefits apart from merely providing food. Indeed, this food function is no longer predominant and the gardeners themselves usually underline the educational and therapeutic roles of the garden and its contributions to creating social links. 
INDEX

Keywords : urban agriculture, family gardens, shared gardens, Abbé Lemire, subsistence agriculture, food, insertion, social, environment, education, leisure, hygienics, Bordeaux Mots-clés : agriculture urbaine, jardins familiaux, jardins partagés, Abbé Lemire, agriculture vivrière, alimentation, insertion, social, environnement, éducation, loisir, hygiénisme, Bordeaux

\section{AUTEUR}

\section{VÉRONIQUE SAINT-GES}

Économiste, INRA, UMR SAD-APT INRA-AgroParisTech veronique.saint-ges@inra.fr 